\title{
Erratum to "Complete Characterization of Trees with Maximal Augmented Zagreb Index"*
}

\author{
Xiaoshi Xiao ${ }^{1}$, Xiuliang Qiu ${ }^{1}$, Wenshui Lin ${ }^{2,3, \dagger}$ \\ ${ }^{1}$ Chengyi University College, Jimei University, \\ Xiamen 361021, China \\ 2 Fujian Key Laboratory of Sensing and Computing for Smart City, \\ Xiamen 361005, China \\ ${ }^{3}$ School of Informatics, Xiamen University, Xiamen 361005, China
}

(Received February 14, 2021)

\begin{abstract}
The augmented Zagreb index $(A Z I)$ has attracted more and more attentions in the past years. Some significant mathematical properties of $A Z I$ were obtained. In particular, Lin et al. [MATCH Commun. Math. Comput. Chem. 83 (2020) 167 recently claimed a complete solution to the problem of characterizing $n$-vertex tree(s) with maximal $A Z I$. In this note we correct some errors in the paper.
\end{abstract}

\section{Introduction}

Let $G=(V, E)$ be a connected simple graphs, where $V=\left\{v_{0}, v_{1}, \ldots, v_{n-1}\right\}$ and $n \geq 3$. $d_{i}=d\left(v_{i}\right)$ will denote the degree of vertex $v_{i}$. The augmented Zagreb index $(A Z I)$ of $G$ is defined [1] as $A Z I(G)=\sum_{v_{i} v_{j} \in E}\left[d_{i} d_{j} /\left(d_{i}+d_{j}-2\right)\right]^{3}$. This index was shown to have the best predicting ability for a variety of physicochemical properties among several tested vertex-degree-based topological indices (see $[2,3]$ ). Hence, this molecular

\footnotetext{
*Supported by the National Natural Science Foundation of China (No. 11771362), the Industry University Research Innovation Fund of Chinese Universities (No. 2019ITA01053), and the Scientic Research Fund of Fujian Provincial Department of Education (No. JAT201032).

${ }^{\dagger}$ Corresponding author. Email address: wslin@xmu.edu.cn
} 
descriptor has attracted more and more attentions in the past years. Some significant mathematical properties of $A Z I$ were obtained. Most known results can be found in the review article [4]. For latest developments after the publication of [4] see [5-7]. In particular, Lin et al. [8] claimed a complete solution to the problem of characterizing $n$ vertex tree(s) with maximal $A Z I$, an open problem proposed by Furtula et al. [1]. That is, the following result was proven in [8].

Theorem 1.1. If $n \geq 19$, then the balanced double star $B D_{n}$ is the unique $n$-vertex tree with maximal $A Z I$.

However, there are some errors in the Case 2 of the proof of Theorem 1.1 in [8]. Since the problem is so elementary, the errors are worth to be corrected.

\section{Preliminaries}

To point out and correct the errors in [8], we have to introduce more notations and results.

Let $h(x, y)=[x y /(x+y-2)]^{3}$ for $x, y \geq 1$ with $x+y \geq 3, l(x, y)=h(x, y)-h(x-1, y)$ for $x \geq 2$ and $y \geq 1$ with $x+y \geq 4$, and $f(x)=(x-2) l(x, 1)+h(x, 1)=(x-1) h(x, 1)-$ $(x-2) h(x-1,1)$ for $x \geq 3$.

\section{Lemma $2.1[9]$.}

(1) $h(x, 1)$ strictly decreases with $x \geq 2$.

(2) $h(x, 2)=8$.

(3) If $y \geq 3$ is fixed, then $h(x, y)$ strictly increases with $x \geq 2$.

Lemma 2.2 [10]. For $x \geq 2$ and $y \geq 1$,

(1) $l(x, 1)(<0)$ strictly increases with $x \geq 3$.

(2) $l(x, 2)=0$ for $x \geq 3$.

(3) If $y \geq 3$ is fixed, then $l(x, y)$ (>0) strictly increases with $2 \leq x \leq y-1$, and strictly decreases with $x \geq y$.

Lemma $2.3[10]$. If $y>x \geq 2$, then $l(x, y)>l(y, x)$. Hence $h(x+1, y-1)>h(x, y)$ if $y \geq x+2 \geq 3$.

Lemma 2.4 [10]. Let $x \geq 3$. Then $f(x)$ strictly increases with $x$, and $-1.25 \leq f(x)<1$.

Besides the above known results, the following two new results are needed.

Lemma 2.5. If $y \geq 7$, then $l(y+1, y+1)>l(y-3, y+1)>l(y-5, y+1)$.

Proof. From Lemma $2.2(3)$ it suffices to show $l(y+1, y+1)>l(y-3, y+1)$. Let $g(x, y)=[(y-x+1) /(2 y-x)]^{3}, x \leq y$. Then $g_{x}{ }^{\prime}(x, y)=3(1-y)(y-x+1)^{2} /(2 y-x)^{4}$, 
and $g_{x}{ }^{\prime \prime}(x, y)=6(x-2)(y-1)(y-x+1) /(2 y-x)^{5}$. Hence $g_{x}{ }^{\prime}(x, y)$ strictly decreases with $x \leq 2$ and strictly increases with $2<x \leq y$, and we have

$$
\begin{aligned}
& l(y+1, y+1)-l(y-3, y+1) \\
= & h(y+1, y+1)-h(y, y+1)-[h(y-3, y+1)-h(y-4, y+1)] \\
= & \frac{[(y+1)(y+1)]^{3}}{(2 y)^{3}}-\frac{[y(y+1)]^{3}}{(2 y-1)^{3}}-\left\{\frac{[(y-3)(y+1)]^{3}}{(2 y-4)^{3}}-\frac{[(y-4)(y+1)]^{3}}{(2 y-5)^{3}}\right\} \\
= & (y+1)^{3}\{g(0, y)-g(1, y)-[g(4, y)-g(5, y)]\} \\
= & (y+1)^{3}\left[g_{x}^{\prime}\left(\xi_{2}, y\right)-g_{x}^{\prime}\left(\xi_{1}, y\right)\right] \\
> & (y+1)^{3}\left[g_{x}^{\prime}(4, y)-g_{x}^{\prime}(0, y)\right] \\
= & 3(y+1)^{3}(y-1)\left[\frac{(y+1)^{2}}{(2 y)^{4}}-\frac{(y-3)^{2}}{(2 y-4)^{4}}\right]
\end{aligned}
$$

where $0<\xi_{1}<1$ and $4<\xi_{2}<5$. It is easily seen that

$$
\begin{aligned}
\frac{(y+1)^{2}}{(2 y)^{4}}-\frac{(y-3)^{2}}{(2 y-4)^{4}}>0 & \Leftrightarrow \frac{y+1}{(2 y)^{2}}>\frac{y-3}{(2 y-4)^{2}} \\
& \Leftrightarrow(y+1)(2 y-4)^{2}>(y-3)(2 y)^{2} \\
& \Leftrightarrow 16>0,
\end{aligned}
$$

and the conclusion holds.

Analogously, we have the following result.

Lemma 2.6. If $y \geq 7$, then $l(y+1, y)>l(y-5, y)$.

Proof. Let $g(x, y)=[(y-x+1) /(2 y-x-1)]^{3}, x \leq y$. Then $g_{x}{ }^{\prime}(x, y)=3(2-y)(y-$ $x+1)^{2} /(2 y-x-1)^{4}$, and $g_{x}{ }^{\prime \prime}(x, y)=6(x-3)(y-2)(y-x+1) /(2 y-x-1)^{5}$. Hence $g_{x}{ }^{\prime}(x, y)$ strictly decreases with $x \leq 3$ and strictly increases with $3<x \leq y$, and we have

$$
\begin{aligned}
& l(y+1, y)-l(y-5, y) \\
= & h(y+1, y)-h(y, y)-[h(y-5, y)-h(y-6, y)] \\
= & \frac{[y(y+1)]^{3}}{(2 y-1)^{3}}-\frac{[y y]^{3}}{(2 y-2)^{3}}-\left\{\frac{[y(y-5)]^{3}}{(2 y-7)^{3}}-\frac{[y(y-6)]^{3}}{(2 y-8)^{3}}\right\} \\
= & y^{3}\{g(0, y)-g(1, y)-[g(6, y)-g(7, y)]\} \\
= & y^{3}\left[g_{x}^{\prime}\left(\xi_{2}, y\right)-g_{x}^{\prime}\left(\xi_{1}, y\right)\right] \\
> & y^{3}\left[g_{x}^{\prime}(6, y)-g_{x}^{\prime}(0, y)\right] \\
= & 3 y^{3}(y-2)\left[\frac{(y+1)^{2}}{(2 y-1)^{4}}-\frac{(y-5)^{2}}{(2 y-7)^{4}}\right]>0,
\end{aligned}
$$

where $0<\xi_{1}<1$ and $6<\xi_{2}<7$. 
Remark. The best possible of Lemma 2.6 may be $l(y+1, y)>l(y-4, y)$ for $y \geq 6$. However, the proof may be difficult, and the same technique used above is not applicable.

\section{Correction to the errors}

For convenience, let $T$ be an $n$-vertex $(n \geq 19)$ tree with maximal $A Z I$. It is known [10] that $A Z I(T) \geq A Z I\left(B D_{n}\right)>n^{3} / 64+n+3$. Let $\pi=\left(z=d_{0}, y=d_{1}, \ldots, x=d_{t}, 1^{n-t-1}\right)$ be the non-increasing degree sequence of $T$. The known results of $\pi$ can be summarized as the following lemma.

Lemma $3.1[8,10] .1 \leq t \leq z=d_{0} \geq 10, d_{1}=d_{2}=\cdots=d_{t-1}=y \geq z-1 \geq 9$, and $3 \leq x=d_{t} \leq y$.

When Lin et al. [8] proved Theorem 1.1, in the Case $2(t \geq 2)$ it was assumed that $h(x-2, y+2)>h(x, y)$ in the Subcase 2.1 and $h(x-2, y+1)>h(x-1, y)$ in the Subcase 2.2. However, they are wrong according to Lemma 2.3. Here we give the correction to the errors.

Correct proof of the Case 2 in the proof of Theorem 1.1 in [8]. Since the conclusion holds for $n \leq 64$ from the computer search results in [10], we assume $n \geq 65$.

Case 1. $x \leq y-5$. Let $u$ be a child (a leaf) of $v_{t}$, and $T_{1}=T-v_{t} u+v_{1} u$. Then from Lemmas $2.2(3)$ and $2.4-2.6$ we have

$$
\begin{aligned}
A Z I\left(T_{1}\right)-A Z I(T)= & h(y+1, z)+h(x-1, z)+(x-2) h(x-1,1)+y h(y+1,1) \\
& -[h(y, z)+h(x, z)+(x-1) h(x, 1)+(y-1) h(y, 1)] \\
= & l(y+1, z)-l(x, z)-f(x)+f(y+1) \\
> & l(y+1, z)-l(y-5, z)>0 .
\end{aligned}
$$

Case 2. $x \geq y-4$. From Lemma 3.1 we have $2 n-2 \geq z+(t-1) y+x+n-t-1 \geq$ $(t+1) y+n-t-5$, which yields $y \leq(n+t+3) /(t+1)$ and $n \geq y(t+1)-t-3 \geq 8 t+6>6 t+6$.

From Lemmas 2.1 and 3.1 we have

$$
\begin{aligned}
A Z I(T) & <(t-1) h(y, y+1)+h(x, y+1)+(n-t-1) h(x, 1) \\
& \leq t h(y, y+1)+(n-3) h(y-4,1) \\
& \leq t h\left(\frac{n+t+3}{t+1}, \frac{n+2 t+4}{t+1}\right)+(n-3) h(1,5) \\
& \leq \frac{t}{8(t+1)^{3}}\left[\frac{(n+t+3)(n+2 t+4)}{n+0.5 t+2.5}\right]^{3}+\frac{125}{64}(n-3)
\end{aligned}
$$




$$
<\frac{t}{8}\left(\frac{n+4 t+4}{t+1}\right)^{3}+2 n \triangleq \eta(t) .
$$

It is easily seen that

$$
\begin{aligned}
\eta^{\prime}(t) & =\frac{1}{8}\left(\frac{n+4 t+4}{t+1}\right)^{3}-\frac{3 \operatorname{tn}}{8} \frac{(n+4 t+4)^{2}}{(t+1)^{4}} \\
& =\frac{(n+4 t+4)^{2}}{8(t+1)^{4}}\left[(1-2 t) n+4(t+1)^{2}\right] \\
& <\frac{(n+4 t+4)^{2}}{8(t+1)^{4}}\left[(1-2 t)(6 t+6)+4(t+1)^{2}\right] \\
& =\frac{(n+4 t+4)^{2}}{8(t+1)^{4}}[(t+1)(-8 t+10)]<0 .
\end{aligned}
$$

That is, $\eta(t)$ strictly decreases with $t \geq 2$, and $A Z I(T)<\eta(2)=(n+12)^{3} / 108+2 n$. Thus from $n \geq 65$ it holds that

$$
\begin{aligned}
A Z I(T)<A Z I\left(B D_{n}\right) & \Leftarrow \frac{n^{3}}{64}+n+3 \geq \frac{(n+12)^{3}}{108}+2 n>0 \\
& \Leftarrow \frac{n^{3}}{64}+3 \geq \frac{(n+12)^{3}}{108}+1.01 n \\
& \Leftrightarrow n \geq 64.9626 .
\end{aligned}
$$

The proof is thus completed.

\section{References}

[1] B. Furtula, A. Graovac, D. Vukičević, Augmented Zagreb index, J. Math. Chem. 48 (2010) 370-380.

[2] I. Gutman, J. Tošović, Testing the quality of molecular structure descriptors. Vertexdegree-based topological indices, J. Serb. Chem. Soc. 78 (2013) 805-810.

[3] I. Gutman, Degree-based topological indices, Croat. Chem. Acta 86 (2013) 351-361.

[4] A. Ali, B. Furtula, I. Gutman, D. Vukičević, Augmented Zagreb index: Extremal results and bounds, MATCH Commun. Math. Comput. Chem. 85 (2021) 211-244.

[5] F. Li, Q. Ye, H. Broersma, R. Ye, Sharp upper bounds for augmented Zagreb index of graphs with fixed parameters, MATCH Commun. Math. Comput. Chem. 85 (2021) $257-274$.

[6] A. Ali, A note on minimal augmented Zagreb index of tricyclic graphs of fixed order, MATCH Commun. Math. Comput. Chem. 85 (2021) 247-256. 
[7] T. Alraqad, A. Ali, H. Saber, Solution of an open problem concerning the augmented Zagreb index and chromatic number of graphs, MATCH Commun. Math. Comput. Chem. 86 (2021) 39-48.

[8] W. Lin, D. Dimitrov, R. Škrekovski, Complete characterization of trees with maximal augmented Zagreb index, MATCH Commun. Math. Comput. Chem. 83 (2020) $167-178$.

[9] Y. Huang, B. Liu, L. Gan, Augmented Zagreb index of connected graphs, MATCH Commun. Math. Comput. Chem. 67 (2012) 483-494.

[10] W. Lin, A. Ali, H. Huang, Z. Wu, J. Chen, On the trees with maximal augmented Zagreb index, IEEE Access 6 (2018) 69335-69341. 